\author{
Н.Д. Панкратова, Г.В. Горелова, В.А. Панкратов \\ Институт прикладного системного анализа КПИ им.Игоря Сикорского
}

\title{
ИНСТРУМЕНТАРИЙ ПЛАНИРОВАНИЯ ПОДЗЕМНОЙ ИНФРАСТРУКТУРЫ МЕГАПОЛИСА НА ОСНОВЕ МЕТОДОЛОГИЙ ПРЕДВИДЕНИЯ И КОГНИТИВНОГО МОДЕЛИРОВАНИЯ
}

\begin{abstract}
Предложен инструментарий моделирования и сценарного анализа планирования развития подземной инфраструктуры мегаполиса в условиях экологических, техногенных и террористических угроз на основе совместного применения методологий предвидения и когнитивного моделирования. Разработка экологизированных и безопасных сценариев планирования развития подземного урбанизированного пространства осуществляется на основе методов теории графов, симплициального анализа, импульсного моделирования.

Ключевые слова: подземная инфраструктура, предвидение, когнитивное моделирование, численная и структурная устойчивости, импульсное моделирование, сценарии.

\author{
Н.Д. Панкратова, Г.В. Горелова, В.А. Панкратов \\ Інститут прикладного системного аналізу КПІ ім. Ігоря Сікорського
}

\section{ІНСТРУМЕНТАРІЙ ПЛАНУВАННЯ ПІДЗЕМНОЇ ІНФРАСТРУКТУРИ МЕГАПОЛІСУ НА ОСНОВІ МЕТОДОЛОГІЙ ПЕРЕДБАЧЕННЯ І КОГНІТИВНОГО МОДЕЛЮВАННЯ}

Запропоновано інструментарій моделювання і сценарного аналізу планування розвитку підземної інфраструктури мегаполісу в умовах екологічних, техногенних і терористичних загроз, що базується на спільному застосуванні методологій передбачення, та когнітивного моделювання. На першому етапі застосовується методологія передбачення, отримані результати використовуються в якості вихідних даних на другому етапі - для когнітивного моделювання, що дозволяс запропонувати науково обгрунтовану стратегію реалізації пріоритетної альтернативи сценарію планування розвитку підземної інфраструктури мегаполісу.

Застосування методології передбачення як процесу прийняття рішень для складних систем із людським фактором, надає можливість побудувати їх бажану поведінку в майбутньому. Такий процес зводиться до залучення методики сценарного аналізу, в межах якої використовуються окремі якісні методи у певній послідовності із встановленням чітко визначених взаємозв'язків між ними, в результаті цього процесу отримаються альтернативи сценаріїв планування розвитку інфраструктури мегаполісу. Для обгрунтованої реалізації альтернативи сценарію залучається когнітивне моделювання, що дозволяс на підставі знання i досвіду побудувати причиннонаслідкові зв'язки, зрозуміти і проаналізувати поведінку складної системи на страте-

Исследования выполнены при частичной поддержки Национального фонда исследования Украины по гранту 2020.01/0247

(C) Панкратова Н.Д., Горелова Г.В., Панкратов В.А., 2020 
гічну перспективу при великій кількості взаємозв'язків і взаємозалежностей. При дослідженні даної проблеми при залученні результатів, отримних в процесі передбачення, будуються когнітивні моделі типу когнітивної карти - знакового оріснтованого графа і функціонального графа у вигляді зваженого знакового орграфа. Для дослідження властивостей когнітивної моделі використовуються методи аналізу структурної стійкості і стійкості до збурень, методи аналізу зв'язності моделі (симпліціального аналіз), методи теорії графів. Для визначення можливого розвитку процесів в складній системі і розробки розвитку сценаріїв планування розвитку ынфраструктури мегаполюсу залучасться модель імпульсного процесу.

Ключові слова: підземна інфраструктура, передбачення, когнітивне моделювання, чисельна і структурна стійкості, імпульсне моделювання, сценарії.

\author{
N.D. Pankratova, G.V. Gorelova, V.A. Pankratov \\ Institute for Applied System Analysis Igor Sikorsky Kyiv Polytechnic Institute
}

\title{
TOOLS FOR PLANNING OF METROPOLITAN UNDERGROUND INFRASTRUCTURE BASED ON FORESIGHT AND COGNITIVE MODELING METHODOLOGIES
}

The tools of modeling and scenario analysis of underground infrastructure of the metropolis development planning in the conditions of ecological, technogenic and terrorist threats, based on joint application of methodologies of foresight and cognitive modeling are offered. At the first stage the foresight methodology is used, the obtained results are used as initial data at the second stage - for cognitive modeling, which allows to offer a scientifically based strategy for implementing a priority alternative of the planning scenario of the underground infrastructure of the metropolis. The application of foresight methodology as a decision-making process for complex systems with the human factor, provides an opportunity to build their desired in the future. This process is included the methods of scenario analysis, which uses certain qualitative methods in a certain sequence with the establishment of clearly defined relationships between them, as a result of this process will be alternative scenarios for planning of the infrastructure metropolis development.

Cognitive modeling is used for a reasonable implementation of the alternative scenario, which allows to build causal relationships on the basis of knowledge and experience, to understand and analyze the behavior of a complex system for a strategic perspective with a large number of relationships and interdependencies. In the study of this problem, using the results obtained in the process of foresight, cognitive models of the cognitive map type are built - a sign-oriented graph and a functional graph in the form of a weighted sign digraph. To study the properties of the cognitive model, methods of analysis of structural stability and resistance to perturbations, methods of model analysis connectivity (simplicial analysis), methods of graph theory are used. To determine the possible of processes development in a complex system and to develop scenarios for the development planning of the megapole infrastructure, the model of the impulse process is involved.

Key words: underground infrastructure, foresight, cognitive modeling, numerical and structural stability, impulse modeling, scenarios.

Введение. Развитие мегаполисов в настоящее время актуализирует проблемы подземной урбанистики. Рост крупных городов является проявлением постоянных исторических закономерностей и ведет не только к постоянному увеличению размеров мегаполисов, но и к существенному усложнению их функционально-пространственной организации, причем во многих случаях 
уже исчерпаны традиционные возможности развития города «вверх и вширь» и начато широкое освоение решения ряда острых проблем, связанных с интенсивным ростом мегаполисов: территориальных, транспортных, энергетических, экологических и др. может быть успешно осуществлено путем строительного освоения городского подземного пространства $[1,2]$.

Миллионы людей полагаются сегодня на подземные коммуникации, которые надежно обеспечивают удобство и комфорт. Эксплуатация новой подземной инфраструктуры стимулирует и поддерживает устойчивое развитие городов, становится его неотъемлемой составляющей $[3,4]$. При этом хорошо спланированная и правильно эксплуатируемая подземная инфраструктура повышает качество жизни, энергетическую эффективность и экологическую безопасность в большей степени, чем аналогичная система на поверхности.

Кардинальные изменения, которые произошли в последние десятилетия в жизни больших городов, требуют научного осмысления новых реалий и наиболее вероятных перспектив их дальнейшего развития. Подземная урбанистика, которая является неотъемлемой составляющей современных мегаполисов, уже вышла за пределы отдельных локальных объектов и становится системным фактором существования крупных городов. Предвидения будущих изменений, соответствующая градостроительный политика, планирование развития мегаполисов должны опираться на надежный научнометодический фундамент, который должен обеспечивать развитие наземной и подземной застройки как одного целого [5-7].

В основу современного подхода к планированию мегаполисов положена системная концепция устойчивого развития города, в пределах которой должны удовлетвориться современные потребности общества без потерь и убытков для грядущих поколений [8]. Важным аспектом устойчивого развития городов является возможность своевременного реагирования на структурно-функциональные и естественные изменения среды и принятия решения по минимизации техногенных и экологических рисков. Эта концепция влияет на масштаб и стратегию многих инженерных проектов и предусматривает изменение традиционного видения локальных задач в сторону направление рассмотрения проектов с позиций крупных естественнотехнических и социальных систем.

Городской подземное пространство может обеспечить трехмерную свободу передвижения людей, материальных, водных и энергетических ресурсов, в частности в малодоступных объектов в плотно застроенных районах города. Сложность освоения подземного пространства крупных городов обусловлена не только необходимостью обоснований структурно-функциональных и пространственных решений, но и переменными свойствами геологической среды, в которой размещаются подземные комплексы, накладывает дополнительные факторы влияния и элементы рисков. Формирование культуры предвидения и управления сопровождения рисков должно стать важной составляющей проектного мышления современного инженера-геостроителя [9-11]. 
Большие города Украины, в частности Киев, Харьков, Днепр, Одесса, Львов активно развивают строительство современных подземных объектов, однако градостроительный потенциал подземного пространства используется пока недостаточно. Проекты подземных сооружений остаются в основном одиночными, локальными и недостаточно связанными между собой и объектами на поверхности, а строительство ведется без стратегического плана развития «подземного города» и научного анализа альтернатив. Существенное отставание по степени использования подземного пространства Киева от группы мировых лидеров (Хельсинки, Лондон, Монреаль, Осака, Пекин, Сингапур, Токио, Шанхай и др.) обусловлено не только вопросами инвестиционного климата, но и слабостью политики территориального развития, недостаточным уровнем законодательного и нормативно-правового обеспечения вопросов собственности объектов геостроительства, отсутствием согласованной концепции и стратегического мастер-плана развития подземной урбанистики столицы и, как следствие, нехваткой обоснованных привлекательных предложений для мировых инвестиционных групп [12-14].

Авторы поставили целью своей работы раскрыть преимущества системной методологии освоения подземного пространства мегаполисов и продемонстрировать возможности синтеза методологий предвидения и когнитивного моднлирования для оценки рисков и предвидения построения альтернатив сценариев развития подземной урбанистики как системы альтернативных разнотипных проектных конфигураций, что может стать научнометодической основой построения стратегического мастер плана «подземного города» и способствовать развитию механизма оценки социальноэкономической эффективности освоения городского подземного пространства мегаполисов.

Постановка задачи. Разрабатываемый инструментарий моделирования и сценарного анализа планирования развития подземной инфраструктуры мегаполиса в условиях экологических, техногенных и террористических угроз базируется на совместном применении методологий предвидения и когнитивного моделирования. Предлагается использовать указанные методологии совместно: на первом этапе применять методологию предвидения, полученные результаты использовать в качестве исходных данных на втором этапе для когнитивного моделирования, что позволяет предложить научно обоснованную стратегию реализации приоритетной альтернативы сценария планирования развития инфраструктуры мегаполиса. Применение методологии предвидения как процесса принятия решений для сложных систем с человеческим фактором, дает возможность построить их желаемое поведение в будущем. Такой процесс сводится к привлечению методологии сценарного анализа, в рамках которой используются определенные методы качественного анализа в определенной последовательности с установлением четко определенных взаимосвязей между ними, в результате этого процесса создаются альтернативы сценариев планирования развития инфраструктуры мегаполиса $[15,16]$. Однако из-за наличия человеческого фактора имеющаяся информа- 
ция имеет ряд характеристик, которые не позволяют использовать сугубо традиционные методы анализа больших объемов информации - данные могут быть неполными, нечеткими, недостоверными, противоречивых. Кроме того, техногенные, экологические, экономические процессы могут приобретать качественные изменения. Прогнозирование их течения как экстраполяции выявленных трендов является не всегда целесообразным, поскольку определенные события могут полностью изменить поведение исследуемой системы, что делает имеющиеся прогнозы недействительными. Преодоление этой проблемы возможно только за счет привлечения знаний, опыта, интуиции экспертов в соответствующих областях. Поэтому целесообразно решение задач предвидения проводить на основе углубленной аналитики слабоструктурированных данных по техногенным, экологическим и экономическим процессам [17]. Сочетание методик и приемов обработки больших объемов неструктурированной информации с методологиями системного и сценарного анализа обеспечит возможность долгосрочного предвидения поведения сложных систем различной природы на основе использования фактов, отношений, показателей, полученных как традиционным для предвидния экспертным оцениванием, так и путем углубленного анализа большого корпуса текстовых документов. Это позволяет адекватно и своевременно разрабатывать и адаптировать стратегии в условиях постоянного роста объемов актуальной информации и изменений в окружающем мире, при этом учитывая существование различного вида неопределенностей и многофакторных степеней и уровней риска, гарантирует устойчивое развитие исследуемой системы в соответствии с условиями развития, избранных в сценариях ключевых технологий и отраслей. Для обоснованной реализации альтернативы сценария привлекается когнитивное моделирование, что позволяет на основании знания и опыта построить причинно-следственные связи, понять и проанализировать поведение сложной системы на стратегическую перспективу при большом количестве взаимосвязей и взаимозависимостей [18].

Формализация методологии когнитивного моделирования. Когнитивное моделирование сложных систем является имитационным моделированием структуры и поведения сложной системы на основе количественных и качественных данных о ней и проводится поэтапно. Основные этапы: 1) разработка когнитивной модели в той или иной математической форме (от когнитивной карты до векторного параметрического функционального графа); 2) исследование свойств модели, на основании которых делается заключение о не противоречии свойств модели свойствам исследуемой сложной системы; 3) сценарный анализ, проводимый путем импульсного моделирования на когнитивной моделе, позволяющий определить возможное развитие ситуаций в системе под воздействием на нее внешних, внутренних возмущений и управляющих решений. Целью таких когнитивных исследований является понимание, объяснение, формальное представление сложной системы (на основе структуризации имеющихся о ней теоретических, практических знаний, статистических и экспертных данных) и порождение новых знаний о системе, 
что необходимо для принятия обоснованных управленческих решений. Разработанная методология [18] и программное обеспечение [19] когнитивного моделирования являются инструментом исследования свойств сложной системы (устойчивости, связности, сложности и др.) и ее поведения [20]. В совокупности с методологией предвидения, применяемой на первом и третьем этапах когнитивного моделирования, результаты исследования служат для разработки рекомендаций по совершенствованию сложной системы, для предложения желаемых стратегий развития системы и предотвращения не желаемых. Это можно считать завершающим этапом исследования.

В исследовании проблемы обоснования выбора пригодности земельного участка для подземного строительства на первом этапе были использованы когнитивные модели типа когнитивной карты - знакового ориентированного графа (1) и функционального графа в виде взвешенного знакового орграфа (3), (4) [18].

$$
G=\langle V, E\rangle,
$$

где $G$ - когнитивная карта, в которой $V-$ концепты, конечное множество вершин когнитивной карты $V_{i} \in V, i=1,2, \ldots k ; E=\left\{e_{i j}\right\}-$ множество дуг $e_{i j}$ графа, $i, j=1,2, \ldots m$, отражают взаимосвязь между вершинами $V_{i}$ и $V_{j}$; влияние $V_{i}$ на $V_{j}$ в изучаемой ситуации может быть положительным $(+1)$, когда увеличение (уменьшение) одного фактора приводит к увеличению (уменьшению) другого, отрицательным (-1), когда увеличение (уменьшение) одного фактора приводит к уменьшению (увеличению) другого, или отсутствовать (0). Когнитивной карте $G$ соответствует квадратная матрица отношений $A_{G}$

$$
A_{G}=\left\{a_{i j}\right\}=\left\{\begin{array}{cccccc}
1, & \text { если } & V_{i} & \text { связано } & c & V_{j} \\
0, & \text { в } & \text { противном } & \text { случае } & &
\end{array} .\right.
$$

Отношение $a_{i j}$ может принимать значение «+1» или «-1». Отношение между переменными (взаимодействие факторов) - это количественное или качественное описание влияния изменения одной переменной на другие в соответствующих вершинах.

Векторный функциональный граф

$$
\Phi=\langle G, X, F(X, E), \theta\rangle,
$$

где $G$ - когнитивная карта; $X$ - множество параметров вершин, $\theta$ пространство параметров вершин; $F(X, E)$ - функционал преобразования дуг. Если

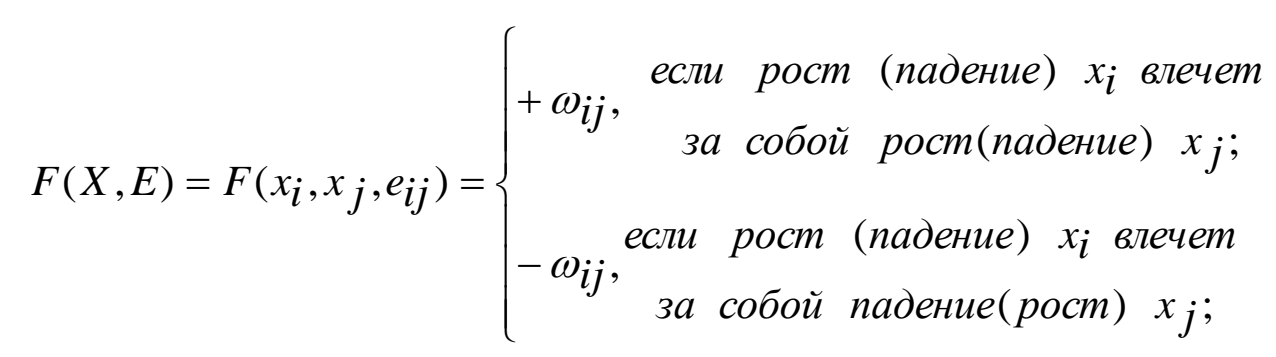

то имеет место взвешенный знаковый орграф, в котором $\varlimsup_{i j}$ - весовой коэффициент. 
На втором этапе когнитивного моделирования для исследования свойств когнитивной модели использовались методы анализа структурной устойчивости и устойчивости к возмущениям [20-22], методы анализа связности модели (симплициальный анализ [23-24]), методы теории графов [22]. Результаты анализа сопоставлялись с имеющейся информацией о подземном строительстве.

На третьем этапе когнитивного моделирования для определения возможного развития процессов в сложной системе и разработки сценариев развития была использована модель импульсного процесса (моделирование распространения возмущений на когнитивных моделях) [22]:

$$
x_{v_{i}}(n+1)=x_{v_{i}}(n)+\sum_{v_{j}: e=e_{i j} \in E}^{k-1} f\left(x_{i}, x_{j}, e_{i j}\right) P_{j}(n)+Q_{v i}(n) \text {, }
$$

где $x(n), x(n+1)$ - величины показателя в вершине $V_{i}$ при шагах имитации в момент $t=n$ и следующим за ним $t=n+1 ; P_{j}(n)-$ импульс, существовавший в вершине $V_{j}$ в момент $t=n ; Q_{V i}(n)=\left\{q_{1}, q_{2}, \ldots, q_{k}\right\}$ - вектор внешних импульсов (возмущающих или управляющих воздействии), вносимых в вершины $V_{i}$ в момент времени $n$.

Анализ результатов когнитивного моделирования обоснования выбора пригодности земельного участка для подземного строительства.

Первый этап. Разработка когнитивной модели. При разработке когнитивной модели был использован принцип последовательного уточнения модели: первоначально была построена когнитивная карта (1), затем на ее основе строится модель взвешенного знакового орграфа (3). Приведем этапы построения когнитивной карты (1).

Первый этап. Построение когнитивной карты G1 - «Подземное строительство». При разработке когнитивной модели удобно представлять проанализированную и систематизированную информацию о вершинах в виде табл.1.

Таблица 1

Вершины когнитивной карты $G 1$

\begin{tabular}{|c|c|c|}
\hline Код & Название вершин & Назначение вершин \\
\hline 1 & 2 & 3 \\
\hline$V_{0}$ & Устойчивость участка Suitability of the site & индикативная, целевая \\
\hline$V_{1}$ & $\begin{array}{l}\text { Уровень динамической нагрузки } \\
\text { The level of dynamic load }\end{array}$ & базовая, возмущающая \\
\hline$V_{2}$ & $\begin{array}{c}\text { Показатель статической нагрузки от поверхност- } \\
\text { ной застройки } \\
\text { The indicator of static load from surface development }\end{array}$ & $\begin{array}{c}\text { базовая, } \\
\text { возмущающая }\end{array}$ \\
\hline$V_{3}$ & $\begin{array}{c}\text { Показатель статической нагрузки окружающей } \\
\text { грунтового массива } \\
\text { The indicator of static load from surface development }\end{array}$ & базовая \\
\hline$V_{4}$ & $\begin{array}{c}\text { Влияние существующих подземных объектов } \\
\text { The impact of existing underground facilities }\end{array}$ & возмущающая \\
\hline
\end{tabular}


ISSN 2074-5893 Питання прикладної математики і математичного моделювання. Випуск 20

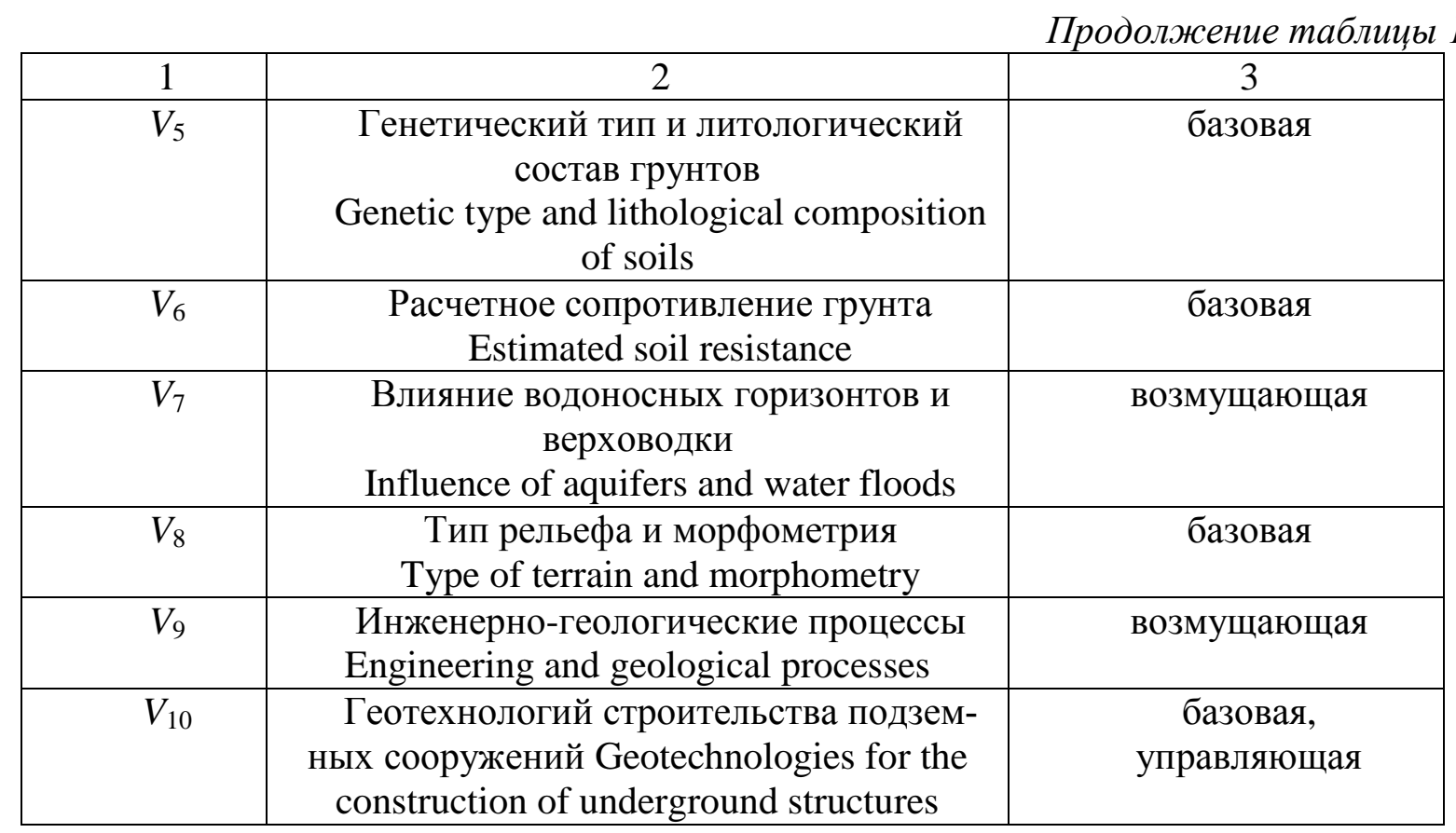

Bторой этап. Анализ свойств когнитивной карты $G 1$. Анализ свойств когнитивной карты $G 1$ включает: определение характеристик графа, анализ путей и циклов, анализ устойчивости к возмущениям и структурной устойчивости. Определение разных путей из вершины в вершину на когнитивной карте позволяет осмысливать, насколько обоснованно определены причинноследственные цепочки исследуемой когнитивной карты «Подземное строительство», в которой заданы основные характеристики предназначаемого для подземного строительства участка. Анализ путей позволяет интерпретировать смысл путей - причинно-следственных цепочек, использовать информацию далее для поддержки принимаемых решений. Кроме того, пути показывает многообразие возможностей достижения целей, их количество может быть большим и далеко не очевидным для лица, принимающего решения. Также становится наглядным, какие вершины включены в каждый путь, «затрагиваются» на этом пути, что дает повод для оценки его желательности или не желательности. На рис.1 приведены примеры выделения путей между вершинами $V_{10}$ (Geotechnologies for the construction of underground structures) и $V_{0}$ (Suitability of the site); всего таких путей 16; на рис.1 изображены один из положительных (нет отрицательных дуг или имеется четное число отрицательных дуг) и один из отрицательных (нечетное число отрицательных дуг) путей.

Анализ циклов когнитивной карты (рис.2) показал, что в этой системе содержится 47 циклов, среди них - 20 циклов положительной (усиливающей) и 27 циклов отрицательной (стабилизирующей) обратной связи. Это свидетельствует о том, что анализируемая система структурно устойчива [22]. Оценка этого факта как «хороший» или «плохой» не однозначна, может 


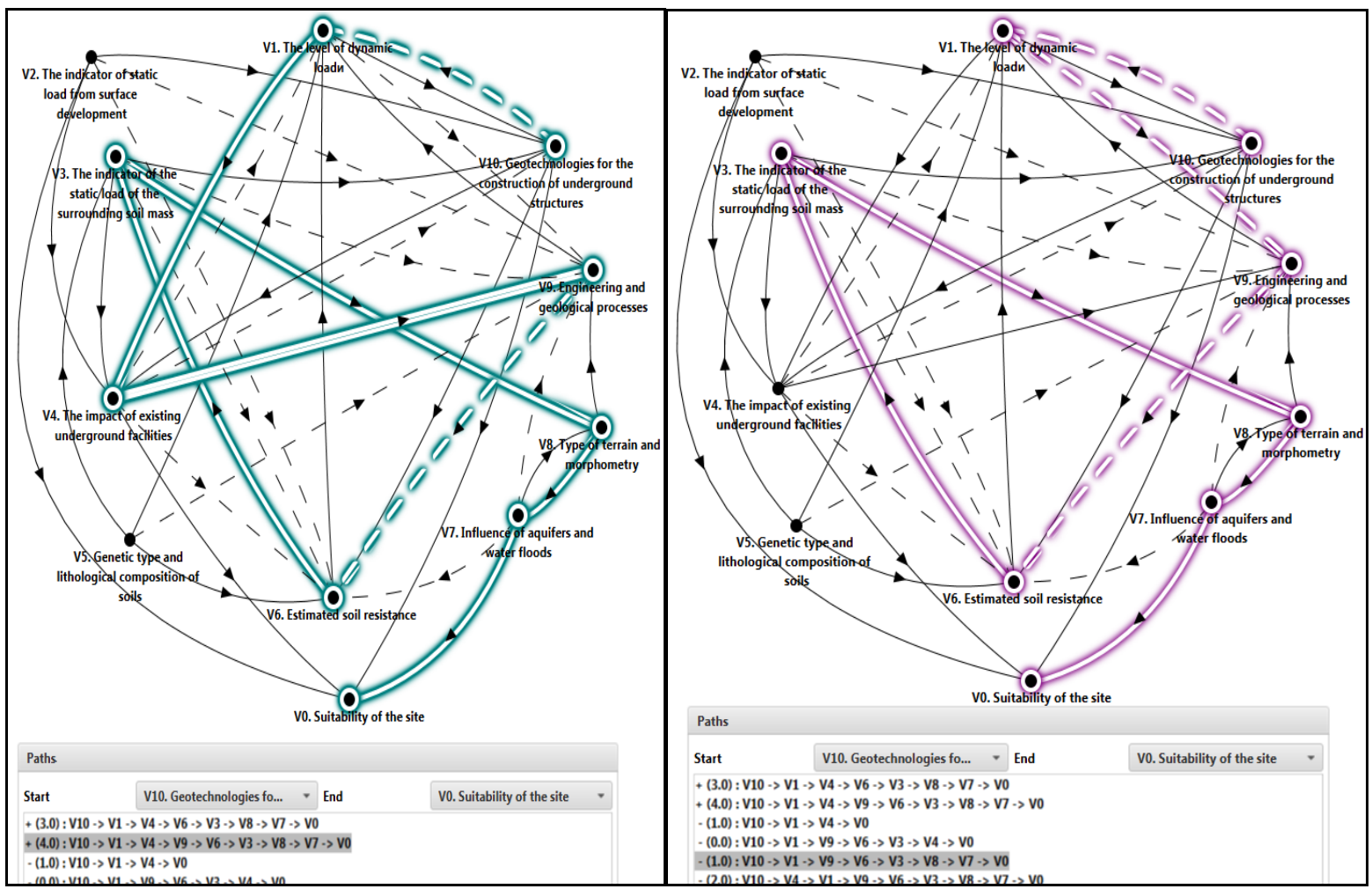

Рис. 1. Пути графа $G_{1}$ из вершины $V_{10}$ к вершине $V_{0}$

зависеть как от целей управления, так и от «предпочтений» ЛПР. который может модернизировать начальную когнитивную карту.

Для анализа устойчивости к возмущениям использован критерий устойчивости $|\mathrm{M}|<1$, где $|\mathrm{M}|-$ это максимальное по модулю собственное число матрицы $R_{G 1}$. Поскольку в данном случае $|\mathrm{M}|=1,9309>1$, то система $G 1$ не устойчива ни к возмущению, ни по начальному значению. Это свидетельствует о том, что малейшие отклонения в вершинах выводят систему из устойчивого состояния. Принятие решения о том, насколько это «хорошо»/«Плохо» в данном случае, требует продолжения анализа и уточнения модели.

Tретий этап. Импульсное моделирование, анализ сценариев. С помощью программной системы CMLS [19] возможно внесение возмущений $Q=\left\{q_{i}\right\}$, $i=1,2, \ldots k$ разной величины (нормированной) в любую из вершин, а также в их комбинацию. Перед началом импульсного моделирования необходимо разработать план вычислительного эксперимента. При внесении возмущений в вершины, ищется ответ на вопрос: «А что будет, если ...?». В процессе импульсного моделирования возможно вносить возмущающие воздействия на любом такте моделирования. Это дает возможность изменять (корректировать) сценарии в модельной динамике, определять воздействия, приближающие процессы к желаемым. Приведем некоторые варианты эксперимента.

Сценарий №1. Пусть геотехнологии строительства подземных сооружений (Geotechnologies for the construction of underground structures) совершенствуются, импульс $q_{10}=+1$ вносится в вершину $V_{10}$, вектор возмущений $Q_{1}=\left\{q_{1}=0 ; q_{2}=0 ; \ldots q_{10}=+1\right\}$. 

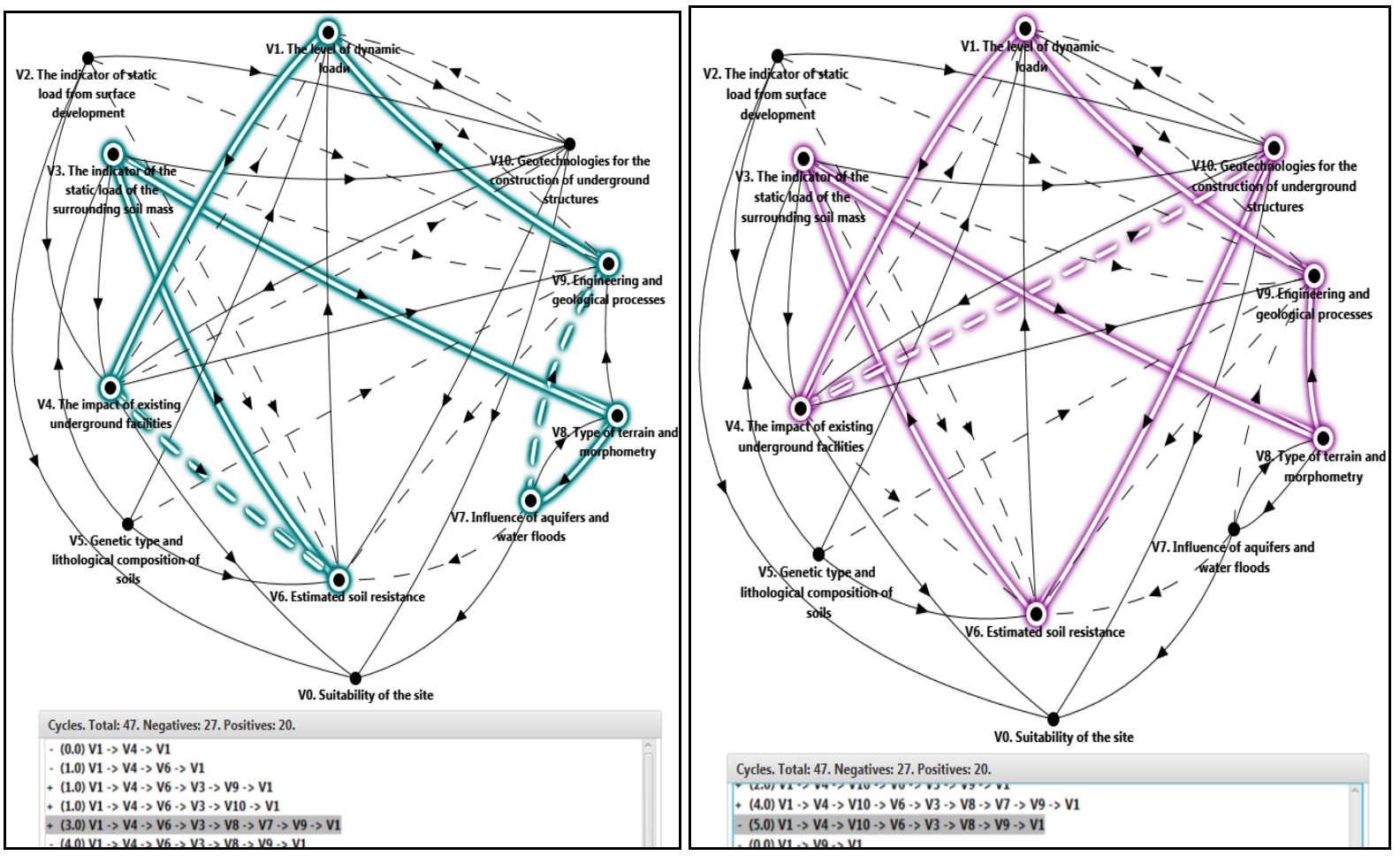

Рис. 2. Исследование циклов на когнитивной карте $\boldsymbol{G}_{\mathbf{1}}$

На рис.3 представлены результаты расчетов импульсных процессов (по формуле (4)), на 10 шагах моделирования, число которых в данном случае можно считать достаточным, т.к. тенденции развития ситуаций в системе уже проявились.

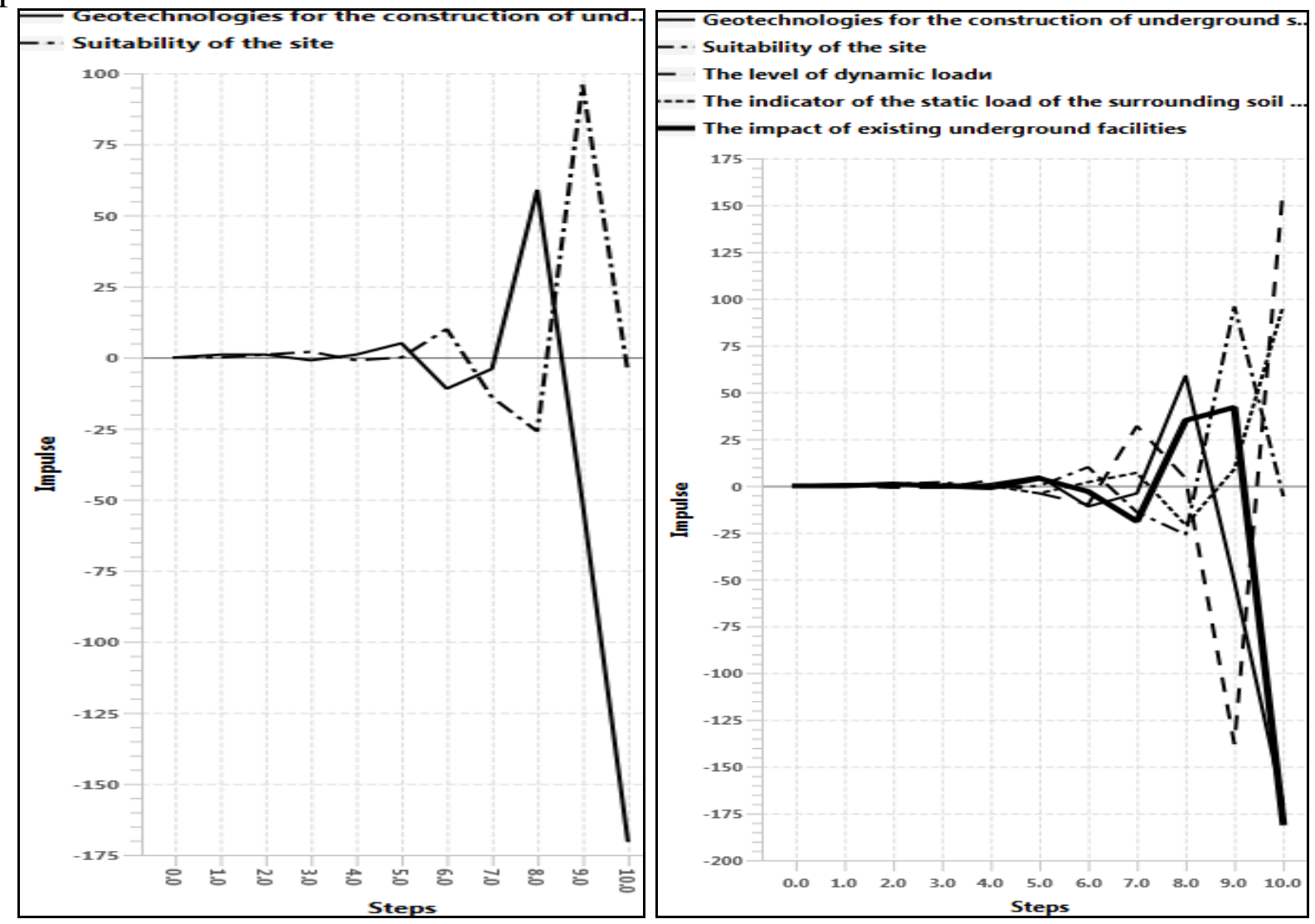

Рис. 3. Графическое представление импульсных процессов по сценарию №1 
Моделирование сценария №1 показало, что в случае модели системы, представленной в виде когнитивной карты $G 1$, процессы в ней будут развиваться в нарастающем колебательном режиме при предполагаемых изменениях в вершине $V_{10}$. Рассмотрим еще один сценарий.

Сценарий №v2. Пусть происходит возбуждение четырех вершин: отрицательное влияние существующих подземных объектов $\left(q_{4}=-1\right)$ и водоносных горизонтов и верховодки $\left(q_{7}=-1\right)$, но инженерно-геологические процессы и геотехнологии строительства подземных сооружений сказываются положительно $\left(q_{9}=+1\right.$ и $\left.q_{10}=+1\right)$; вектор возмущений $Q_{2}=\left\{0 ; \ldots q_{4}=-1 ; . . q_{7}=-1 ; \ldots q_{9}=+1\right.$; $\left.q_{10}=+1\right\}$. Результаты расчетов представлены в на рис. 4 .

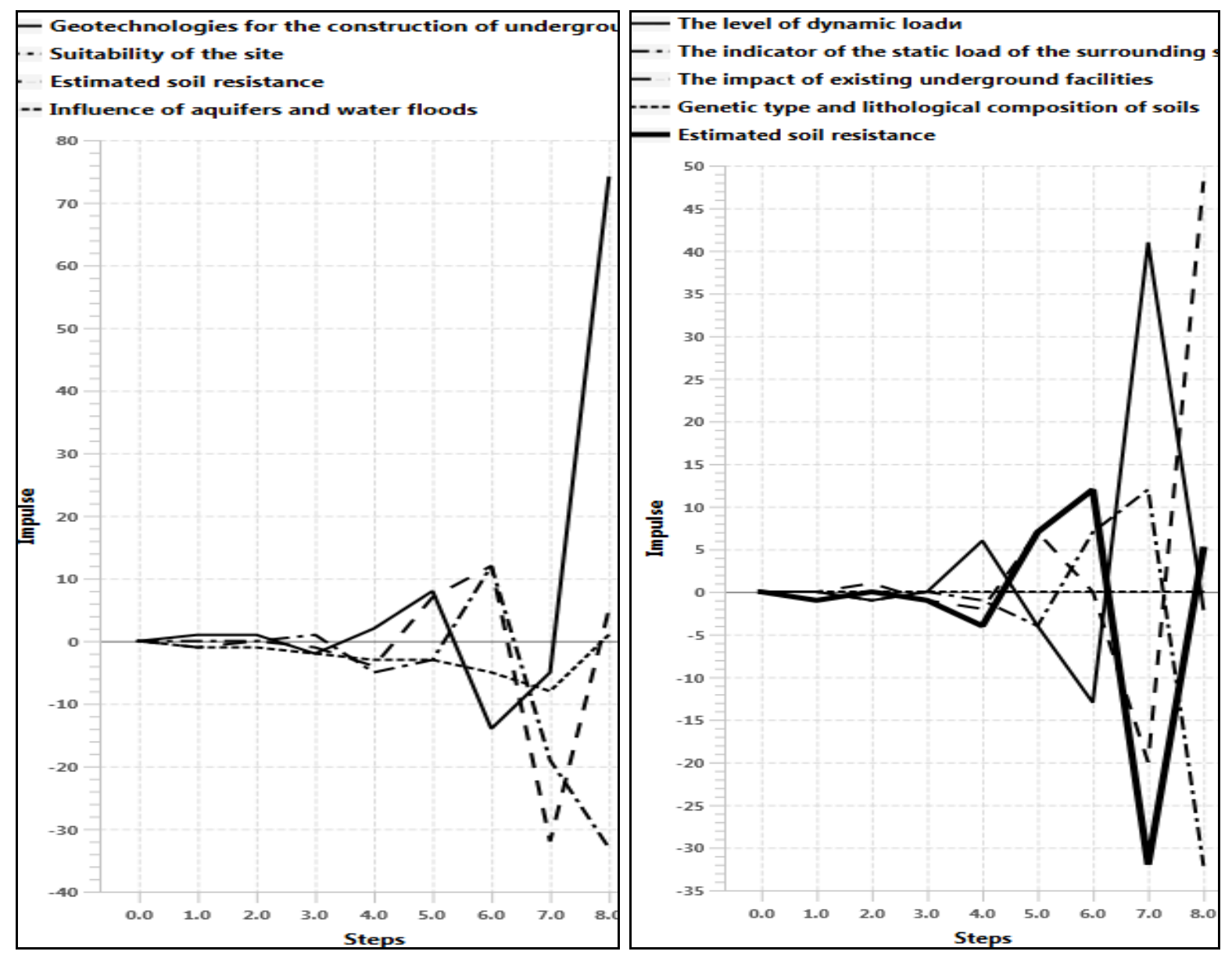

Рис. 4. Графическое представление импульсных процессов по сценарию №2

Анализируя результаты сценарного моделирования №2 видим, что наблюдается такой же характер процессов, как и по сценарию №1. Модель $G 1$ оказывается импульсно неустойчивой, что следует также из предыдущих результатов исследования когнитивной карты $G 1$ на устойчивость к возмущениям. Но модель $G 1$ устойчива структурно, а ее неустойчивость к возмущениям может быть вызвана тем, что все отношения $a_{i j}$ одинаковы, равны единице. Это может не соответствовать действительности. В продолжение исследований был проведен экспертный опрос, с помощью которого были установлены весовые коэффициенты дуг и проведены вычислительные экс- 
перименты, в результате которых построены желаемые сценарии, параметры которых передаются заказчику.

Заключение. Разработанный инструментарий моделирования и сценарного анализа планирования развития подземной инфраструктуры мегаполиса в условиях экологических, техногенных и террористических угроз, базирующийся на совместном использовании методологий предвидения и когнитивного моделирования, применяется для исследования объектов подземного строительства с целью выбора желаемых сценариев их дальнейшего развития. Моделирование сценариев возможных процессов развития событий в анализируемой сложной системе осуществляется под воздействием различных внутренних и внешних возмущений и управляющих импульсных воздействий. Результаты проведенного когнитивного моделирования позволяют судить о том, что когнитивные модели, систематизирующие и структурирующие различную информацию о системе подземного строительства, соответствуют реальной системе и могут быть использованы для планирования возможных процессов ситуаций в системе под воздействием различных возмущающих и управляющих факторов. Разработанный авторский программный комплекс CMLS позволяет в процессе импульсного моделирования и анализа полученных результатов вводить управляющие или возбуждающие воздействия на любом этапе моделирования. Это позволяет изменять (корректировать) сценарии в динамике создания модели, определять эффекты, приближающие процессы к желаемым. Разработанная методология и инструментальные средства позволили совместить оценку воздействий и взаимосвязей геологических факторов техногенного и структурно-функционального типов для исследования строительства подземных объектов в целях обеспечения безопасности и качества жизни человека.

\section{Библиографические ссылки}

1. Kartosiya, B.A. Developing underground space of large cities. New tendencies [Text]. Mining information-analytical bulletin. 1. - 2015. - P.615-629.

2. Gilbert, P.H. Underground Engineering for Sustainble Urban Development [Text]. Washington: The National Academies Press. - 2013.

3. Sterling, R. Sustainability issues for underground spaces in urban areas. Proceedings of ICE [Text]. / R. Sterling, H. Admiraal, N. Bobylev, H. Parker, J.P. Godard, I. Vähäaho, X. Shi, and T. Hanamura // Urban Design and Planning. Volume 165, Issue 4. - December 2012. - P. 241-254

4. Owen, C. L., Bezerra C. Evolutionary Structured Planning. A Computer-Supported Methodology for the Conceptual Planning Proces [Text] / C. L. Owen, C. Bezerra In JS Gero (ed.). Artificial Intelligence in Design'00. Kluwer Academic Publishers, Dordrecht, - 2000. P. 287-307.

5. Gayko, G.I. Developing underground space in the concept of sustainable development for large cities [Text]. - Geotechnologies. 1. - 2018. - P.60-64.

6. Pankratova, N. System approach to planning urban underground development [Text] / N. Pankratova, I. Savchenko, H. Haiko, V. Kravets // Int. Journal "Information Content and Processing" 6(1) - 2019.- P. 3-17. 
7. Pankratova, N. Problems of Megapolises Underground Space System Planning [Text] / N. Pankratova, G. Gayko, V. Kravets, I. Savchenko // Journal of Automation and Information Sciences 48,4. - 2016. - P. 32-38.

8. Vernadsky, V. I. Biosphere and noosphere [Text]. - M . Iris Press - 2012.

9. Konykhov, S.S. Systematization of approaches for developing underground city space [Text]. - Vestnik MGSU. 4. - 2010. - P. 56-61.

10. Resin V.I., Popkov Yu.S. Large cities development in conditions of transition economy (system approach) [Text]. - Knizhnyi dom "LIBROKOM", Moscow. - 2013.

11. Bondarik, G.K. General theory of engineering (physical) geology [Text]. - Nedra, Moscow, 1981.

12. Vähäaho, I. Underground space planning in Helsinki[Text] // Journal of Rock Mechanics and Geotechnical Engineering. 6. - 2014. - P. 387-398.

13. Langley, P. Cognitive architectures: Research issues and challenges [Text] / P. Langley, J.E. Laird, S. // Rogers Cognitive Systems Research, vol.10 (2), -2009. - P.141-160.

14. Powell, J. H. An application of a network-based futures method to strategic business planning [Text] // Journal of the Operational Research Society. №48. - 997. - P.857-872.

15. Gorelova, G.V. Scientific Foresight and Cognitive Modeling of Socio-Economic Systems [Text] / G.V. Gorelova, N. D. Pankratova, 18 th IFAC Conference on Technology, Culture and International Stability, TECIS-2018, IFAC. Volume 51, Issue 30. - 2018. - P. 145-149.

16. Pankratova, N. D. Strategy for the Study of Interregional Economic and Social Exchange Based on Foresight and Cognitive Modeling Methodologies [Text] / N.D. Pankratova, G.V. Gorelova, V.A. Pankratov // Proceedings of the 8th International Conference on "Mathematics. Information Technologies. Education, Shatsk, Ukraine, June 2-4. - 2019. P. 136-141.

17. Pankratova, N. Assessment of situations in the field of social disasters basing on the methodology of foresight and textual analytics [Text] / Pankratova N., Savastiyanov V. // Proceedings of the 2019 IEEE Second International Conference IEEE UKRCON. -2019. P. 1207-1210.

18. Innovative development of socio-economic systems based on foresight and cognitive modelling methodologies. In editors Gorelova G.V., Pankratova N.D. Kiev, Naukova Dumka 2015.

19. Program for cognitive modeling and analysis of socio-economic systems at the regional level. Certificate of state registration of computer programs N 2018661506. -2018 .

20. Згуровский, М.3. Стратегия инновационного развития региона на основе синтеза методологий предвидения и когнитивного моделирования [Текст] / М.З. Згуровский, B.A. Панкратов //System Research\&Informatio Technologies. № 2. - 2014. - C. 7 - 17.

21. Casti, J. Connectivity, Complexity, and Catastrophe in Large-scale Systems [Text] / J. Casti, A Wiley // Interscience Publication International Institute for Applied Systems Analysis. JOHN WILEY and SONS. Chichester - New York - Brisbane -Toronto. -1979.

22. Roberts, F. Graph Theory and its Applications to Problems of Society [Text]. - Society for Industrial and Applied Mathematics, Philadelphia. - 1978.

23. Atkin, R. H.: Combinatorial Connectivies in Social Systems. An Application of Simplicial Complex Structures to the Study of Large Organisations [Text] // Interdisciplinary Systems Research. - 1997.

24. Atkin, R. H. Polyhedral Dynamics and the Geometry of Systems [Text] / R. H. Atkin, Casti J. RR-77-International Institute for Applied Systems Analysis, Laxenburg, Austria. -1977.

Поступила в редколлегию 16.10. 2020. 